\title{
Light Propagation and Radiative Exciton Transport in Two- Dimensional Layered Perovskite Microwires
}

Yangzi Zhengl, Rounak Naphade ${ }^{2}$, Navendu Mondal $^{1}$, Osman M. Bakr ${ }^{2}$,Omar F. Mohammed ${ }^{2}$, Yuri N. Gartstein ${ }^{1}$, Anton V. Malko ${ }^{1}$

${ }^{1}$ Department of Physics, The University of Texas at Dallas, Richardson, Texas 75080, United

States; ${ }^{2}$ Advanced Membranes and Porous Materials Center (AMPMC), KAUST Catalysis

Center (KCC), Division of Physical Sciences and Engineering, King Abdullah University of Science and Technology, Thuwal 23955-6900, Kingdom of Saudi Arabia.

KEYWORDS: 2D hybrid perovskites, layered-perovskite microwire structure, time-resolved spectroscopy, radiation trapping, self-absorption, photon recycling 


\begin{abstract}
Layered quasi-two-dimensional perovskites are promising candidates for optoelectronic applications exhibiting excitons with high emission quantum yields, high stability and ease of bandgap tunability. Here, we demonstrate a long-range $(\sim 100 \mu \mathrm{m})$ exciton transfer in layered perovskite structure $(\mathrm{en})_{4} \mathrm{~Pb}_{2} \mathrm{Br}_{9} \cdot 3 \mathrm{Br}$ with the ethylene diammonium (en) as a spacer that takes place via the reabsorption of emitted photons. Using the two-objectives setup, we directly map the spatio-temporal dynamics of photoluminescence (PL) from perovskite microwires that reveals a clear spectroscopic signatures of photon recycling: the appearance of PL emission rise times and corresponding elongation of the PL decay as a function of separation distance between the excitation and emission locations. We further show that a kinetic model based on the photonmediated mechanism of the lateral exciton propagation indeed successfully describes all the salient features of the experimental data and gives an independent assessment of the radiative efficiency of the exciton recombination. Our demonstration points out the possibility of judiciously exploiting light management strategies for future high-performance optoelectronic devices with layered perovskite structures.
\end{abstract}


Metal-halide perovskite materials have seen an enormous rise in popularity, proliferating into several research areas of significance including solar cells, lasers, light emitting diodes (LEDs), light sources and radiation detectors owing to facile synthesis methods, defect tolerance, tunable bandgaps, remarkable transport properties and high photoluminescence quantum yield (PLQY). ${ }^{1,2,3,4,5,6,7,8,9}$ Perovskite-based LEDs attract considerable attention due to inherent ability to generate highly pure emission with broad tunability and efficiency. Majority of the explored optoelectronic devices utilize perovskites with the three-dimensional (3D) electronic structure, e.g.

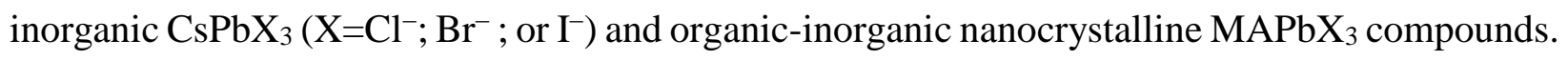
From the photophysics perspective, such bulk materials feature nearly free delocalized charge carriers and the light emission resulting from the slow bimolecular electron-hole recombination. As photovoltaic and LED devices may focus on alternative emission properties, it should be noted that faster radiative recombination with density-independent PLQY can be achieved in systems, where the emission takes place from tighter-bound electron-hole pairs, or excitons. Along these lines, perovskite nanocrystals (PNCs) of various compositions and quantum confinement have been employed, with reported LED efficiencies of $13 \% .{ }^{10,11}$ and higher. While possessing high PLQY under optical excitation, PNC-based LEDs however suffer from low charge mobilities stemming from insulating ligands that are necessary for their synthesis, thus affecting the electrical excitation routes.

Layered hybrid quasi-two-dimensional (2D) perovskites offer a viable approach to efficient light emissive configurations. Compared to the 3D counterparts, they have much larger exciton binding energies $(\gtrsim 0.1 \mathrm{eV})$ caused by the quantum and dielectric confinement in similarity to $2 \mathrm{D}$ atomic layers and semiconductor multi-quantum wells (MQWs). They have superior moisture and chemical stability while manipulation of interlayer cation moieties allows for various degrees of 
electronic transport between the layers. ${ }^{12}$ For instance, the Ruddlesden-Popper phase (RPP) perovskites have a general composition $R_{2} A_{n-1} B_{n} X_{3 n+1}$, $(A$-central cation, $B$-metal, $X$ - halide $)$ where a group of $n$ layers of connected inorganic $\left[\mathrm{BX}_{6}\right]^{4-}$ octahedral sheets are separated from each other by larger organic cations $R$, essentially representing a MQW structure. Already first of these structures demonstrated emission efficacy on par with or better than their 3D counterparts..$^{13,14,15}$

Characterization and performance of photovoltaic, laser and LED materials are affected by specifics of light emission, propagation and absorption in the comprising waveguiding structures. Repetitive re-absorption and re-emission of light (photon recycling) is an important process for photon management strategies in high-performance optoelectronic devices. The role of light management is particularly well-known for ultrahigh-efficiency photovoltaics, where it was in fact illustrated by advancing GaAs solar performance to record $28.3 \%$ efficiency.${ }^{16}$ In recent years, photon reabsorption in perovskites has also garnered substantial attention. ${ }^{17,18,19,20,21}$ By providing a mechanism of long-range spatial propagation of (photo-) excitations, reabsorption can influence the measurements of other propagation modalities such as charge carrier and exciton diffusion, as well as the measurements of dynamics (lifetime) and PL emission lineshapes. While photon recycling in 3D perovskite structures was extensively addressed, its exploration in 2D counterparts has been quite limited..$^{22}$

Here, we explore and decipher the photoexcitation propagation dynamics in microwires made out of highly emissive compound (en) $)_{4} \mathrm{~Pb}_{2} \mathrm{Br} 9 \cdot 3 \mathrm{Br}$ (thereafter termed as MAen20) with the ethylene diammonium (en) as a spacer, which can be compared to to $n=1$ RPP layered perovskites. Using time and spectrally resolved PL dynamics measurements with a two-objectives setup, we directly track the spatio-temporal evolution of emissive photoexcitations within the microwires to 
the lateral distances that exceed $100 \mu \mathrm{m}$. We observe the appearance of PL emission rise times and corresponding elongation of the PL decay as a function of separation distance $s$ between excitation and emission locations that are clear signatures of the photon recycling. We further demonstrate that a kinetic model based on the photon-mediated mechanism of the lateral exciton propagation indeed successfully describes all the salient features of the experimental data and reveals a high intrinsic radiative efficiency of the exciton recombination in this layered perovskite material.

Sample Preparation, Optical Characterization and Experimental Setup for Microwire Samples. Bulk crystals of MAen20 forming microflake structures shown in Figure 1 were prepared using a modified acid cooling approach. As described in the Supporting Information (SI), the synthesis was slightly modified from the original work of Lemmerer and Billing. ${ }^{23}$ The complete synthesis procedure and characterizations including powder X-ray diffraction (PXRD) to confirm the purity of the perovskite phase, and ${ }^{1} \mathrm{H}$ NMR and ${ }^{13} \mathrm{C}$ NMR to confirm the presence of only one organic cation i.e. $\left(\mathrm{en}^{2+}\right)$ was reported elsewhere. ${ }^{24}$ Samples with microwire morphology were physically cleaved from the bulk structures. As illustrated in Figure S1 of the SI, such microwires can reach hundreds of microns in length and have a typical transverse size in the range of 10-30 $\mu \mathrm{m}$. The
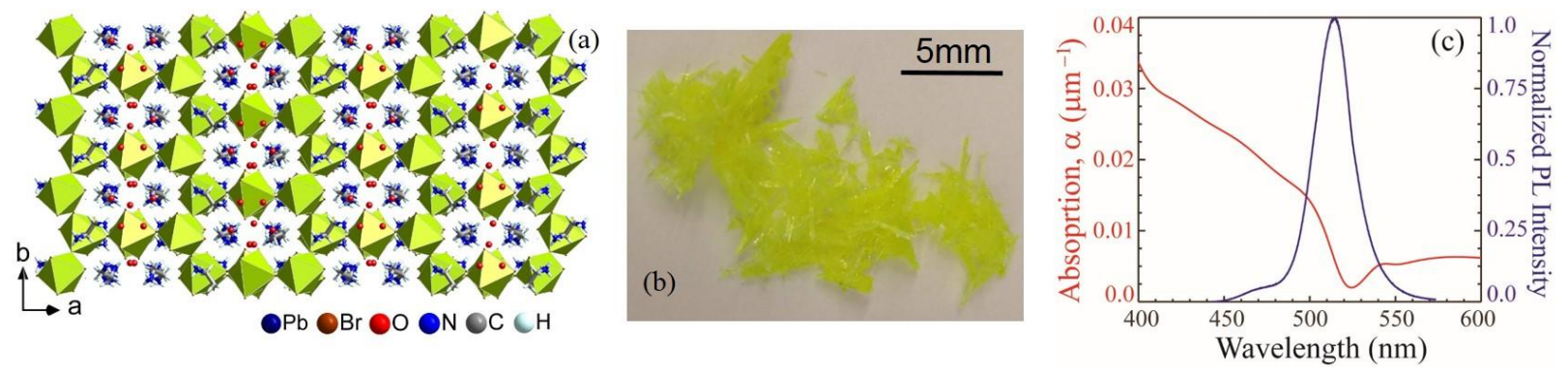

Figure 1. (a) Crystal structure of the MAen20 compound. (b) Optical microphotograph of the microflakes under ambient illumination. (c) Linear absorption and PL spectra of an individual MAen20 microwire. 
crystal structure of the compound is shown in Figure 1 along with absorption/emission spectra of individual microwires. While the motif of the compound exhibits a ribbon-like structure, ${ }^{23}$ the ribbons arrange themselves in strict $2 \mathrm{D}$ layers separated by spacers. It is imporatnt to note that microwire samples are not monocrystals. Even though intrinsically the MAen20 compound possesses anisotropic structure shown in Figure 1(a), this anisotropy is expected to be present only at relatively short (likely sub-micron) spatial scales. On longer-range spatial scales, this anisotropy is lost due to structural defects and random orientations of the crystalline domains arising both during the growth and physical cleaving. The absence of long-range crystalline uniformity can be already seen in the bulk material shown in Figure S1(a), which is further amplified by cleaving "across" domains with different orientations. In addition, Figure S1(b) showing green PL that propagates inside the microwire (and thus illustrating the waveguiding) underlines numerous small domains and structural defects. From the light absorption/propagation point of view, we therefore expect that the microwires can be adequately considered "amorphous" (isotropic) on the spatial scales of relevance to this work.

To study photon recycling effects, we employed the double objective excitation/collection scheme as described in Ref. [19]. In brief, excitation laser light at $800 \mathrm{~nm}$ (two-photon absorption to minimize the surface absorption) is focused via a low magnification objective on the top surface of the microwire while another, a high numerical aperture objective is scanned along the bottom surface. PL signal is recorded as a function of the lateral separation $s$ between the objectives. The use of microwire morphology (where one dimension of the sample is much larger than the others) amplifies the emission waveguiding assistance to accurately measure the signals from remote locations up to and exceeding $100 \mu \mathrm{m}$. Schematics of the double objective configuration are shown 
in Figure 2a. We note that the linear absorption coefficient of an individual microwire in Figure 1c was also measured in the two-objective geometry.

\section{Results and Discussion.}

In our recent publication, we studied light propagation and recycling in $3 \mathrm{D} \mathrm{CsPbBr}_{3}$ microwires. ${ }^{19}$ The primary excitations in such configuration were delocalized electrons and holes leading to observed PL emission lifetimes to be on the order of hundreds of ns. On the other hand, PL
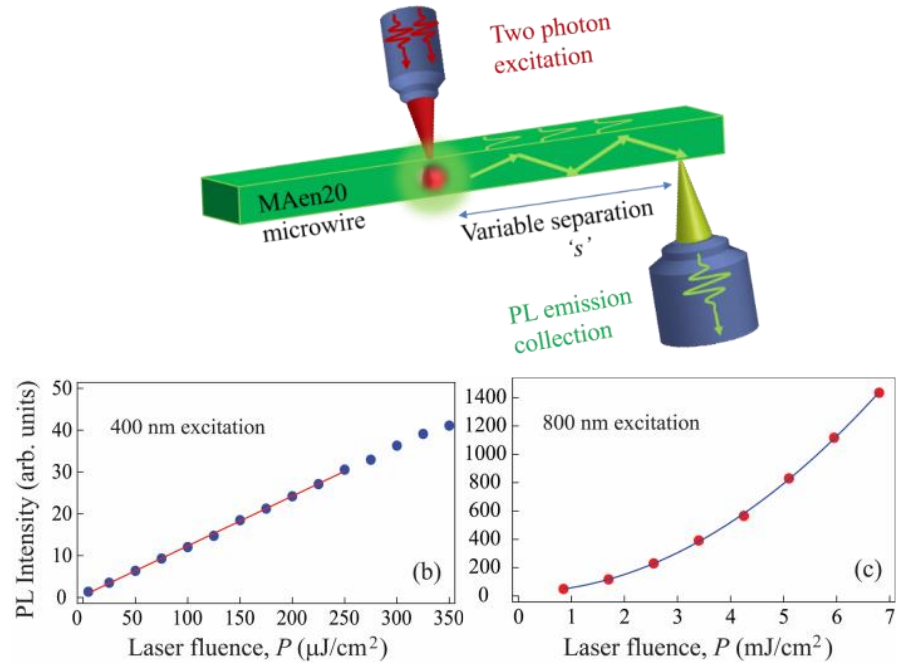

Figure 2. (a) Schematics of the two-objectives setup with variable distance $s$ between PL excitation and collection spots. Power dependence of PL with 1-photon excitation (b) and with 2-photon excitation (c), fitted with linear and parabolic curves, respectively. emission in the compound under study is expected to take place from tighterbound excitonic states that are localized within nanometer-thick layers, potentially affecting photon recycling dynamics and appearance, with lifetimes on the order of nanoseconds. To confirm the nature of the emission sources in MAen20 perovskite waveguides (that is,

excitons vs. bimolecular electron-hole recombination), we performed measurements of PL intensity as a function of the excitation laser fluence $P$. In these experiments we used both onephoton excitation (excitation wavelength $\lambda_{1}=400 \mathrm{~nm}$ ) and two-photon excitation $\left(\lambda_{2}=800 \mathrm{~nm}\right)$. With one-photon excitation the concentration of photo-generated excitons $n_{\text {exc }} \propto P$, with twophoton excitation, however, it would scale as $n_{\text {exc }} \propto P^{2}$. The resulting PL due to radiative recombination of excitons should therefore scale as $\propto P$ or $\propto P^{2}$, respectively. (We do not discuss here high fluences $P$, at which saturation or nonlinear decay processes become relevant.) This is 
indeed the behavior we observed as can be seen in Figure $2(\mathbf{b , c})$, in agreement with the excitonic origin of the emission in 2D MAen20. In contrast, for nearly free electron-hole excitations, it would be concentration $n$ of photoexcited electrons and $p=n$ of holes that would behave as $n \propto P$ (onephoton excitation) or $n \propto P^{2}$ (two-photon excitation). The PL intensity resulting from their bimolecular recombination should then follow $n \cdot p \propto P^{2}$ or $n \cdot p \propto P^{4}$, respectively. These dependences were in fact found in our measurements on $3 \mathrm{D} \mathrm{CsPbBr} 3$ microwires. ${ }^{19}$ We note that from the photophysical perspective, what matters here is the excitonic nature of the emission rather than detailed microscopic structure of excitons; our observations in this paper are thus expected to be applicable to other layered (more generally, lower-dimensional) perovskites.

Figure 3 presents PL spectra and dynamics taken at various separation distances $s$ between excitation $\left(\lambda_{2}=800 \mathrm{~nm}\right)$ and collection spots along an individual MAen20 microwire. We used twophoton excitation scheme to minimize the surface reabsorption or/and surface trapping effects and provide uniform excitation profile through the microwire depth. ${ }^{25}$ The original PL emission is centered at $\sim 520 \mathrm{~nm}$ (corresponding to $s=0 \mu \mathrm{m}$ ) and continuously shifts towards longer wavelengths as the separation is increased, reaching $\sim 525 \mathrm{~nm}$ for $s \sim 100 \mu \mathrm{m}$. In discussing the observed spectral shift of PL at large distances, one needs to distinguish it from the well-known effect of the shift of the spectral content of the propagating light due to the spectral dependence of the medium absorption coefficient. In the geometry of our samples and measurements, this effect would be of relevance only for relatively very short (likely up to several microns) distances between the excitation and observation spots. The exciton emitters at large distances, however, are all positioned spatially equivalently with respect to the observation objective that would thus be measuring the spectral content of their emission. If the PL linewidth was broadened only homogeneously, the PL spectrum would then be the same for different emitters. Under the 

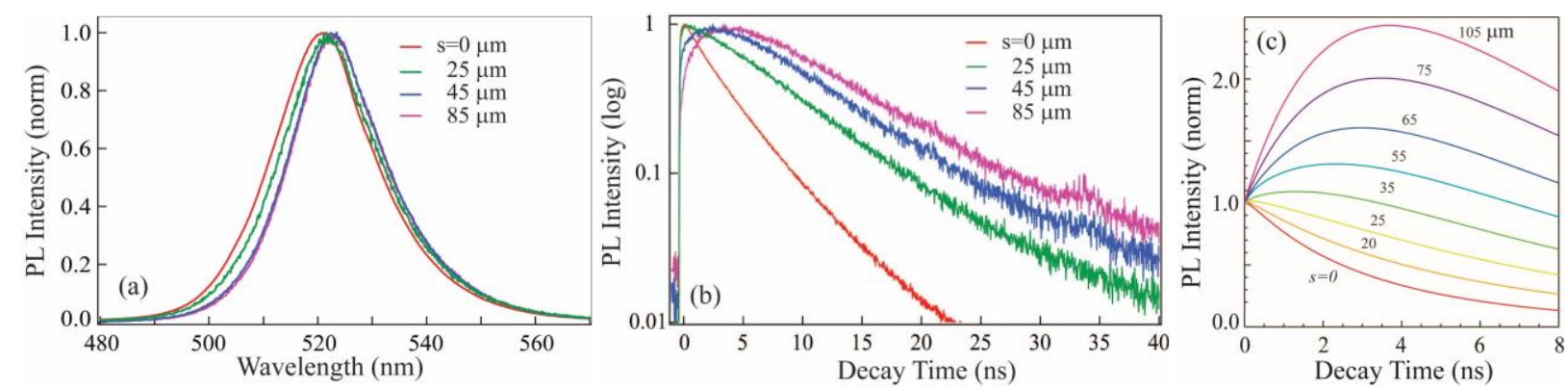

Figure 3: (a) PL spectra; (b) PL decay traces at different excitation/collection separation distances. (c) Fitted PL dynamics at early times, from the early times of the dynamics presented in (b). Data taken at $\mathrm{P}=3.5 \mathrm{~mJ} / \mathrm{cm}^{2}$ fluence of $800 \mathrm{~nm}$ excitation pump.

disorder-induced inhomogeneous contribution to the broadening, subsequent acts of re-absorption and re-emission, on the other hand, result in the gradual shift to lower energy exciton emitters (socalled spectral diffusion towards lower energy emitters in various inhomogeneous systems). How much of such spectral shift would occur evidently depends on the number of re-emission events and the amount of disorder. As we observe relatively small shifts in the current sample, it may be related to the small number of self-absorption/reemission events taking place within the studied separations $s$. Indeed, in our prior work on photon recycling in a different perovskite material with a larger absorption coefficient, similar PL emission shifts were found more substantial in magnitude. ${ }^{19}$ Much more decisive and quantitative determination of the recycling phenomena is given by the signatures in PL dynamics. Figure 3b shows representative behavior of raw PL traces for various separation distances. As outlined, the directly excited PL $(s=0)$ has a short emission lifetime $\tau \sim 4-5 \mathrm{~ns}$, reflecting increased radiative recombination rates of tightly bound excitons. As we increase separation distance, PL decay times progressively increase, reaching $\sim 10 \mathrm{~ns}$ at longer separations. To accurately analyze and represent PL dynamics, we fitted the experimental decay traces with 2-3 exponential functions, fully accounting for the observed rise and fall kinetics in a noise-free format (full set of raw experimental dynamics is shown in Figure S2). Figure 3c depicts early-time PL traces using fitted functions, normalized at the end (zero time) of the excitation laser 
pulse. We clearly observe evolution of the rise times, unequivocally indicating influx of excitations into the distant regions of the sample. As discussed in more detail below, we ascribe this influx to the re-absorption of light emitted from the laser-excited spot and waveguided to the remote regions.

We now proceed with a quantitative analysis of the PL data corresponding to Figure 3(b,c). After the initial excitation, we model the time evolution of PL collected at different distances $s$ from the excitation spot as a function of the delay time $t$. For our thin microwires with the transverse size $\sim 10 \mu \mathrm{m}$, two-photon excitation takes place nearly uniformly across the microwire. A convenient practical framework then is to discuss the spatio-temporal variations $n_{\mathrm{ex}}(x, t)$ of exciton density as a function of just spatial coordinate $x$ along the microwire. Different mechanisms can contribute to the spatial transport of excitons, distinguished in our discussion as either short-range or longrange. The short-range mechanisms would provide for the exciton diffusion and include transport via the diffusion of electron- and hole-constituents or via the near electric fields of the oscillating exciton transition dipole (similar to dipole-dipole energy transfer in molecular systems). The exciton diffusion length $l \sim \sqrt{D \tau}$ is determined by both the effective diffusion coefficient $D$ and lifetime $\tau$ and is expected to be well below 1 micron. As an example, in our previous work on 3D perovskite $\mathrm{CsPbBr}_{3}$ microwires, the diffusion length for charge carriers was estimated to be no more than a fraction of a micron for lifetimes much longer than the PL lifetime $\tau$ observed in the current case of MAen20 microwires. In another example, a recent publication has shown exciton diffusion length not to exceed several hundreds on nanometers in similar $2 \mathrm{D}$ perovskite layers. ${ }^{26}$ Such diffusion lengths are clearly irrelevant for the energy transfer from the excitation spot to PL sources at distances of tens to $100 \mu \mathrm{m}$ away over our time scale $\tau$ of several nanoseconds. Therefore, the observed decay kinetics at such long-range distances and fast transfer is mediated 
by actual photons (that is, by the long-range radiative electromagnetic fields of the oscillating exciton transition dipole), which are initially emitted at the excitation spot and then re-absorbed far away in the microwire leading to PL from that remote location. We thus expect that the salient effects of the reabsorption on the spatio-temporal evolution of the exciton population $n_{\mathrm{ex}}(x, t)$ may be rationalized within the following "minimal-model" kinetic framework:

$$
\frac{\partial n_{\mathrm{ex}}(x, t)}{\partial t}=-\gamma n_{\mathrm{ex}}(x, t)+\eta \gamma \int K\left(x-x^{\prime}\right) n_{\mathrm{ex}}\left(x^{\prime}, t\right) d x^{\prime}
$$

Aimed to emphasize the dominant role of the photon mediation, Eq.(1) explicitly and intentionally neglects the diffusion term. The first term in its r.h.s. describes the exciton decay with rate constant $\gamma$ that would lead to the ordinary mono-exponential behavior $n_{\mathrm{ex}}(x, t) \propto \exp (-\gamma t)$ if not for the exciton generation represented by the second term. This latter term describes the exciton generation at spatial location $x$ due to the absorption of photons emitted by excitons recombining at other spatial locations $x^{\prime}$ and contributing according to spatial kernel $K\left(x-x^{\prime}\right)$. A single dimensionless parameter $0 \leq \eta \leq 1$ defines the efficiency of photon emission upon exciton decay, $\eta=1$ would correspond to purely radiative recombination. Kinetic equation (1) allows one to derive the time-dependent density profile evolution starting from the initial profile $n_{\mathrm{ex}}(x, 0)$. In addition to reabsorption, the emitted photons can also escape the microwire thus determining, as per spatial kernel $L\left(x-x^{\prime}\right)$, the photoluminescence detected at spatial location $x$ :

$$
\operatorname{PL}(x, t) \propto \int L\left(x-x^{\prime}\right) n_{\mathrm{ex}}\left(x^{\prime}, t\right) d x^{\prime}
$$

Equations (1) and (2) assume sufficiently long microwires and translationally invariant kernels, which are then normalized as

$$
\int_{-\infty}^{\infty}[K(x)+L(x)] d x=1 .
$$


Figure 4(a) schematically shows representative trajectories of emitted photons that would be contributing to kernels $K$ and $L$, these trajectories are distinguished by angle $\theta$ with respect to the microwire axis. Depending on this angle, some of the trajectories exhibit total internal reflection from the microwire walls and thus contribute only to kernel $K$, while the others to both $K$ and $L$, in correspondence with reflection coefficient $R(\theta)$. In the SI, we describe the derivation of these kernels as suitable for our purposes here. Consistently with the picture of random orientations of
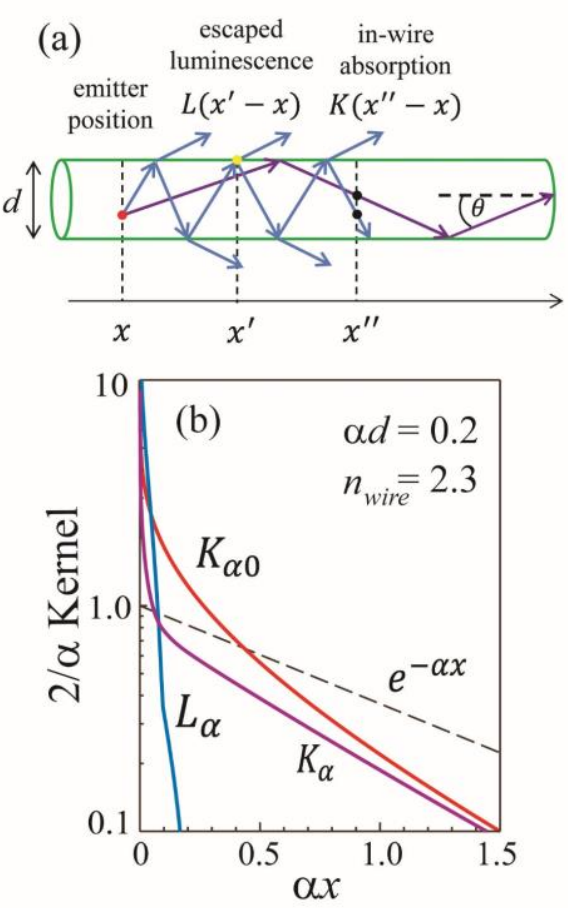

Figure 4. (a) Schematically, examples of photon trajectories originating from emission (red dot) in the cross-section at location $x$ and either leaving the microwire (yellow dot) at location $x^{\prime}$ or absorbed (black dots) at location $x^{\prime \prime}$. (b) Illustration of the spatial behavior of various kernels as described in the text. crystalline parts of the microwire discussed above, the model assumes overall isotropic optical behavior. If all photons were reflected from the walls, that is, if $R(\theta)$ were equal to 1 for all angles, the resulting kernel would be

$$
K_{\alpha 0}(x)=\frac{\alpha}{2} \int_{0}^{\pi / 2} d \theta \frac{\sin \theta}{\cos \theta} e^{-\alpha|x| / \cos \theta},
$$

a familiar benchmark expression from the theory of reabsorption employed for applications in a traditional geometry with the planar air-medium interface, ${ }^{27}$ it satisfies $\int_{-\infty}^{\infty} K_{\alpha 0}(x) d x=1$. Using the picture of "unfolded" photon trajectories, Figure S3, the derivation in SI provides for a simple analytic adaptation of (4) to take into account the escape of emitted photons upon multiple reflection/refraction events for the microwire of transverse size $d$ resulting in 


$$
K_{\alpha}(x)=\frac{\alpha}{2} \int_{0}^{\pi / 2} d \theta \frac{\sin \theta}{\cos \theta} e^{-\alpha|x| / \cos \theta} f\left(\frac{|x| \tan \theta}{d}\right)
$$

and corresponding

$$
L_{\alpha}(x)=-\frac{1}{2 d} \int_{0}^{\pi / 2} d \theta \frac{\sin ^{2} \theta}{\cos \theta} e^{-\alpha|x| / \cos \theta} f^{\prime}\left(\frac{|x| \tan \theta}{d}\right) .
$$

Expressions (4)-(6) are written for a single linear absorption coefficient $\alpha$ in the wire medium and feature function $f(z)$ and its derivative $f^{\prime}(z)=d f / d z$ that is defined as follows. For $0 \leq z \leq 1$, $f(z)=1-[1-R(\theta)] z$, and for larger arguments $z: f(z+1)=R(\theta) f(z)$. As shown in the SI, the value of $z$ in integrals (5) and (6) relates to the number of the reflection/refraction events. Figure 4(b) exemplifies the functional behavior of kernels (5) and (6) for the example of the wire size $d=0.2 / \alpha$ and reflection coefficient $R(\theta)$ corresponding to the interface between air and the wire medium with refraction index $n_{\text {wire }}=2.3$, averaged over emitted light polarizations. These kernels are compared to benchmark (4) as well as to the exponential attenuation with the same linear absorption $\alpha$. Further elaboration on the spatial behavior of the kernels is achieved by accounting for the (normalized) spectral distribution $g(\lambda)$ of the emission wavelength $\lambda$ and the subsequent spread of the wavelength-dependent absorption coefficient $\alpha(\lambda)$ :

$$
K(x)=\int K_{\alpha(\lambda)}(x) g(\lambda) d \lambda, \quad L(x)=\int L_{\alpha(\lambda)}(x) g(\lambda) d \lambda
$$



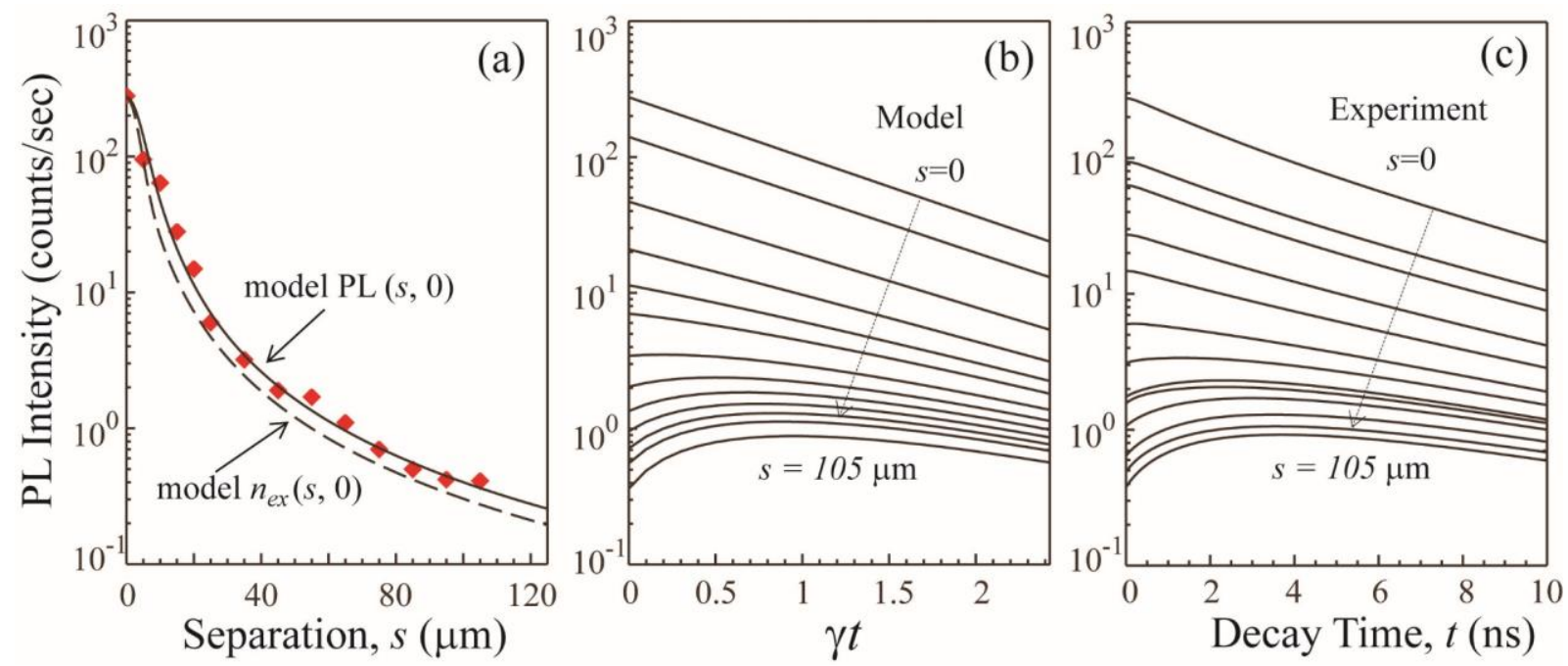

Figure 5. (a) The experimentally measured PL counts at zero delay times (red diamonds) and their model approximation. (b) $\operatorname{PL}(s, t)$ decay traces as functions of dimensionless parameter $\gamma t$, they have been computed per equations (1) and (2) for a set of distances $s$ with parameter $\eta=0.53$. (c) Experimental PL counts as functions of delay time for the same set of separation distances $s$. All panels have the same abscissa axis units explicitly shown for panel (a).

These wavelength-averaged kernels (7) have been evaluated based on the actually measured linear absorption spectrum that is shown in Figure 1(c) and further used for modeling the PL decay traces for the specific microwire sample used in the experiment. Figure 5a displays the initial exciton density $n_{\text {exc }}(s, 0)$ profile that would correspond to the observed initial photoluminescence spatial distribution $\operatorname{PL}(s, 0)$ as a function of distance $s$ from the center of the laser excitation spot. Figure 5(b) shows the results for time-dependent PL traces $\operatorname{PL}(s, t)$ for a series of separation distances between $s=0$ and $s=105 \mu \mathrm{m}$ simulated according to equations (1) and (2) with the emission efficiency parameter $\eta=0.53$. These model results are evidently in a very good match with experimental decay traces for the same series of separation distances shown in Figure 5(c). This match is not supposed to be interpreted as accurate determination of parameter $\eta$. In fact, close values of $\eta$ would also provide satisfying visual comparisons. Instead, we stress that simulations with markedly smaller or larger values of the efficiency parameter $\eta$ show clear deviations from 
the experimentally observed behavior, see Figure S4. Comparing the actual time axis range in Figure 5(c) with the range of the dimensionless parameter $\gamma t$ in Figure 5(b), we can extract the effective exciton life time as $1 / \gamma \cong 4$ ns. Reassuringly, of this value of lifetime and PLQY $~ 50 \%$ derived from the comparison of the experimental and modelling data turn out to be consistent with the room-temperature PL data analysis in MAen20 compound presented in our earlier publication. ${ }^{24}$ In this regard, we note that measurements of the photon recycling can thus be used for an independent assessment of the intrinsic efficiency $(\eta)$ of radiative recombination, particularly when other types of measurements on macroscopic samples are difficult or confounded.

In conclusion, we used direct spatio-temporal spectroscopic probes to unambiguously identify the reabsorption of emitted photons in the layered perovskite compound MAen20 as an efficient mechanism of long-range spatial propagation of excitons. With high intrinsic efficiencies of radiative exciton recombination in $2 \mathrm{D}$ metal-halide perovskites, our demonstration points out the possibility to judiciously design the device structures by exploiting light management strategies. It is known that the layered perovskite structures are more robust with respect to moisture degradation and photobleaching. Moreover, a large variety of organic cations available for layer separation/isolation allows for precise control of the charge funneling and transfer, defect engineering and stability optimization. ${ }^{28}$ Combining these beneficial features with photon management should enable further enhancement of the performance of optoelectronic devices on their basis, particularly for solid-state light emitting applications. 


\section{ASSOCIATED CONTENT}

Supporting Information. Details of perovskite compound synthesis, microphotographs of bulk and microwire samples, original PL lifetime traces, model derivation and additional theoretical figures are provided in supporting figures. This information is available free of charge at ACS publications website http://pubs.acs.org/.

\section{AUTHOR INFORMATION}

\section{Corresponding Author}

*Email: anton.malko@utdallas.edu

\section{Funding Sources}

The work at UT Dallas was supported by the U.S. Department of Energy, Office of Basic Energy Sciences, Division of Materials Sciences and Engineering under Award No. DE-SC0010697.

\section{Notes}

The authors declare no competing financial interest.

\section{REFERENCES:}

${ }^{1}$ Tan, Z.-K.; Moghaddam, R. S.; Lai, M. L.; Docampo, P.; Higler, R.; Descher, F.; Price, M.; Sadhanala, A.; Pazos, L. M.; Credgington, D.; Hanusch, F.; Bein, T.; Snaith, H. J.; Friend, R. H. Bright light Emitting Diodes on Organometal Halide Perovskite. Nat. Nanotech. 2014, 9, 687-692. 
${ }^{2}$ Cho, H.; Jeong, S.-H.; Park, M.-H.; Kim, Y.-H.; Wolf, C.; Lee, C.-L.; Heo, J. H.; Sadhanala, A.; Myoung, N.; Yoo, S.; Im, S. H.; Friend, R. H.; Lee. T.-W. Overcoming the Electroluminescence Efficiency Limitations of Perovskite Light-Emitting Diodes. Science, 2015, $350,1222-1225$.

${ }^{3}$ Dong, H.; Zhang, C.; Liu, X.; Yao, J.; Zhao, Y. S. Materials chemistry and engineering in metal halide perovskite lasers. Chemical Society Reviews, 2020, 49(3), 951-982.

${ }^{4}$ Veldhuis, S. A.; Boix, P. P.; Yantara, N.; Li, M.; Sum, T. C.; Mathews, N.; Mhaisalkar, S. G. Perovskite materials for light-emitting diodes and lasers. Advanced materials, 2016, 28(32), 68046834.

${ }^{5}$ Zhang, L.; Yang, X.; Jiang, Q.; Wang, P.; Yin, Z.; Zhang, X.; Tan, H.; Yang, Y.; Wei, M.; Sutherland, B. R.; Sargent, E. H.; You, J., Ultra-bright and highly efficient inorganic based perovskite light-emitting diodes. Nature Communications, 2017, 8 (1), 15640.

${ }^{6}$ Zhang, T.; Wang, F.; Zhang, P.; Wang, Y.; Chen, H.; Li, J.; Wu, J.; Chen, L.; Chen, Z. D.; Li, S., Low-temperature processed inorganic perovskites for flexible detectors with a broadband photoresponse. Nanoscale, 2019, 11 (6), 2871-2877

${ }^{7}$ Zhang, Y.; Sun, R.; Ou, X.; Fu, K.; Chen, Q.; Ding, Y.; Xu, L.; Liu, L.; Han, Y.; Malko, A. V.; Liu, X.; Yang, H.; Bakr, O. M.; Liu, H.; Mohammed, O. F. Metal Halide Perovskite Nanosheets for X-ray High-Resolution Scintillation Imaging Screens. ACS Nano 2019, 13, 2520-2525.

${ }^{8}$ Alsalloum, A. Y.; Turedi, B.; Zheng, X.; Mitra, S.; Zhumekenov, A. A.; Lee, K. J.; Maity, P.; Gereige, I.; Alsaggaf, A.; Roqan, I. S.; Mohammed, O. F.; Bakr, O. M. Low-Temperature Crystallization Enables 21.9\% Efficient Single-Crystal $\mathrm{MAPbI}_{3}$ Inverted Perovskite Solar Cells. ACS Energy Lett. 2020, 5, 657-662 
${ }^{9}$ Xu, W.; Hu, Q.; Bai, S.; Bao, C.; Miao, Y.; Yuan, Z.; Borzda, T.; Barker, A. J.; Tyukalova, E.;

Hu, Z.; Kawecki, M.; Wang, H.; Yan, Z.; Liu, X.; S, X.; Uvdal, K.; Fahlman, M.; Zhang, W.; Duchamp, M.; Liu, L.-M.; Petrozza, A.; Wang, J.; Liu, L.-M.; Huang, W.; Gao, F. Rational Molecular Passivation for High-Performance Perovskite Light-Emitting Diodes. Nat. Phot. 2019, $13,418-424$.

${ }^{10}$ Li, G.; Rivarola, F. W. R.; Davis, N. J. L. K.; Bai, S.; Jellicoe, T. C.; de la Pena, F.; Hou, S.; Ducati, C.; Gao, F.; Friend, R. H.; Greenham, N. C.; Tan, Z. -K. Highly Efficient Perovskite Nanocrystal Light-Emitting Diodes Enabled by a Universal Crosslinking Method. Adv. Mat. 2016, $28,3528-3534$.

${ }^{11}$ Yan, F.; Xing, J.; Xing, G.; Quan, L.; Tan, S. T.; Zhao, J.; Su, R.; Zhang, L.; Chen, S.; Zhao, Y.; Huan, A.; Sargent, E. H.; Xiong, Q.; Demir, H. V. Highly Efficient Visible Colloidal LeadHalide Perovskite Nanocrystal Light-Emitting Diodes. Nano Lett. 2018, 18, 3157-3164.

${ }^{12}$ Yin, J.; Maity, P.; Naphade, R.; Cheng, B.; He, J.-H.; Bakr, O. M.; Bredas, J.-L.; Mohammed, O. F. Tuning Hot Carrier Dynamics by Dielectric Confinement in Two-Dimensional Hybrid Perovskite Crystals. ACS Nano 2019, 13, 12621-12629.

${ }^{13}$ Wang, N.; Cheng, L.; Ge, R.; Zhang, S.; Miao, Y.; Zou, W.; Yi, C.; Sun, Y.; Cao, Y.; Yang, R.; Wei, Y.; Guo, Q.; Ke, Y.; Yu, M.; Jin, Y.; Liu, Y.; Ding, Q.; Di, D.; Yang, L.; Xing, G.; Tian, H.; Jin, C.; Gao, F.; Friend, R. H.; Wang, J.; Huang, W. Perovskite light-emitting diodes based on solution-processed self-organized multiple quantum wells. Nature Photonics, 2016, 10 (11), 699-704.

${ }^{14}$ Yang, X.; Zhang, X.; Deng, J.; Chu, Z.; Jiang, Q.; Meng, J.; Wang, P.; Zhang, L.; Yin, Z.; You, J., Efficient green light-emitting diodes based on quasi-two-dimensional composition and phase engineered perovskite with surface passivation. Nature Communications, 2018, 9 (1), 570. 
${ }^{15}$ Cortecchia, D.; Yin, J.; Petrozza, A.; Soci, C. White Light Emission in Low-Dimensional Perovskites. J. Mater. Chem. C. 2019, 7, 4956-4969.

${ }^{16}$ Miller, O. D.; Yablonovitch, E.; Kurtz, S. R. Strong internal and external luminescence as solar cells approach the Shockley-Quisser efficiency limit”, IEEE J. Photovoltaics, 2012, 2, 303-311.

${ }^{17}$ Pazos-Outón, L. M.; Szumilo, M.; Lamboll, R.; Richter, J. M.; Crespo-Quesada, M.; AbdiJalebi, M.; Beeson, H. J.; Vrućinić, M.; Alsari, M.; Snaith, H. J.; Ehrler, B.; Friend, R. H.; Deschler, F., Photon recycling in lead iodide perovskite solar cells. Science, 2016, 351 (6280), 1430-1433.

${ }^{18}$ Fang, Y.; Wei, H.; Dong, Q.; Huang, J., Quantification of re-absorption and re-emission processes to determine photon recycling efficiency in perovskite single crystals. Nature Communications, 2017, 8 (1), 14417.

${ }^{19}$ Dursun, I.; Zheng, Y.; Guo, T.; De Bastiani, M.; Turedi, B.; Sinatra, L.; Haque, M. A.; Sun, B.; Zhumekenov, A. A.; Saidaminov, M. I.; García de Arquer, F. P.; Sargent, E. H.; Wu, T.; Gartstein, Y. N.; Bakr, O. M.; Mohammed, O. F.; Malko, A. V., Efficient Photon Recycling and Radiation Trapping in Cesium Lead Halide Perovskite Waveguides. ACS Energy Letters, 2018, 3 (7), 1492-1498.

${ }^{20}$ Brenes, R.; Laitz, M.; Jean, J.; deQuilettes, D. W.; Bulovic, V. Benefit from Photon Recycling at the Maximum Power Point of State-of-the-Art Perovskite Solar Cells. Phys. Rev. Appl. 2019, $12,014017$.

${ }^{21}$ Bowman, A. R.; Anaya, M.; Greenham, N. C.; Stranks, S. D. Quantifying Photon Recycling in Solar Cells and Light-Emitting Diodes: Absorption and Emission are Always Key. Phys. Rev. Lett. 2020, 125, 067401. 
${ }^{22}$ Cho, C.; Zhao, B.; Tainter, G. D.; Lee, J. -Y.; Friend, R. H.; Di, D.; Deschler, F.; Greenham, N. C. The Role of Photon Recycling in Perovskite Light-Emitting Diodes. Nat. Comm. 2020, 11, 611.

${ }^{23}$ Lemmerer, A. and Billing, D. G. Lead Halide Inorganic-Organic Hybrids Incorporating Diammonium Cations, CrystEngComm, 2012, 14, 1954-1966.

${ }^{24}$ Mondal, N.; Naphade, R.; Zhou, X.; Zheng, Y.; Lee, K.; Gereige, I.; Al-Saggaf, A.; Bakr, O. M.; Mohammed, O. F.; Gartstein, Y. G.; Malko, A. V. Dynamical Interconversion Between Excitons and Geminate Charge Pairs in Two-Dimensional Perovskite Layers Described by Onsager-Braun Model, J. Phys. Chem. Lett. 2020, 11, 1112-1119.

${ }^{25}$ Alarousu, E.; El-Zohry, A. M.; Yin, J.; Zhumekenov, A. A.; Yang, C.; Alhabshi, E.; Gereige, I.; AlSaggaf, A.; Malko, A. V.; Bakr, O. M.; Mohammed O. F. Ultralong Radiative States in Hybrid Perovskite Crystals: Compositions for Submillimeter Diffusion Lengths. J. Phys. Chem. Lett. 2017, $8,4386-4390$.

${ }^{26}$ Deng, S.; Shi, E.; Yuan, L.; Jin, L.; Dou, L.; Huang, L. Long-Range Exciton Transport and Slow Annihilation in Two-Dimensional Hybrid Perovskites, Nat. Comm. 2020, 11, 664.

${ }^{27}$ Agranovich, V. M.; Galanin, M. D. Electronic Excitation Energy Transfer in Condensed Matter. North-Holland: Amsterdam, New York, 1983.

${ }^{28}$ El-Ballouli, A. O.; Bakr, O. M. and Mohammed, O. F. Structurally Tunable Two-Dimensional Layered Perovskites: From Confinement and Enhanced Charge Transport to Prolonged Hot Carrier Cooling Dynamics, J. Phys. Chem. Lett., 2020, 11, 5705-5718. 